Research Article

\title{
Transition to Targeted Therapies Improved the Prognosis and Increased the Utilization of Medical Treatments among Patients with Synchronous Metastatic Renal Cell Cancer
}

\author{
Lauri Laru, Hanna Ronkainen, and Markku H. Vaarala \\ Department of Surgery, Medical Research Center Oulu, Oulu University Hospital, University of Oulu, P.O. Box 21, 90029 OYS, \\ Oulu, Finland
}

Correspondence should be addressed to Markku H. Vaarala; markku.vaarala@oulu.fi

Received 15 May 2021; Accepted 3 August 2021; Published 13 August 2021

Academic Editor: Arcangelo Picciariello

Copyright (c) 2021 Lauri Laru et al. This is an open access article distributed under the Creative Commons Attribution License, which permits unrestricted use, distribution, and reproduction in any medium, provided the original work is properly cited.

Since the introduction of targeted therapies (TTs) for metastatic renal cell cancer (mRCC) in 2005, a limited amount of epidemiological data on efficacy of modern drug therapies for synchronous mRCC has been published. We present a comprehensive nationwide cohort including all cases of primarily metastasized renal cell cancer among adults diagnosed between 2005 and 2010 , based on data from the Finnish Cancer Registry and patient records from treating hospitals. Applied treatment protocols and survival outcomes were analyzed. A total of 977 patients were included in the analysis; 499 patients were diagnosed between 2005 and 2007 and 478 patients were diagnosed between 2008 and 2010. The median overall survival (OS) was 8.80 months (95\% confidence interval (CI): 7.60-10.02). The median OS of the patients diagnosed at the latter era was significantly better (11.1; $95 \%$ CI: 8.8-13.4 vs. 7.0; 95\% CI: 5.7-8.3 months, $p \leq 0.001)$. A total number of 524 (53.8\%) patients received drug therapy. Altogether, TTs including tyrosine kinase inhibitors, mammalian target of rapamycin inhibitors (mTORi), and vascular endothelial growth factor inhibitor covered 331 (63.2\%) of first-line treatments, whereas interferon and its combinations with chemotherapy were used for $186(35.5 \%)$ patients. The median OS rates for TT and interferon as first-line therapy groups were 19.9 (16.9-22.8) and 14.9 (12.3-17.4) months, respectively. The OS for patients who did not receive drug therapy after cytoreductive nephrectomy was dismal. We found that the OS estimate of mRCC patients in Finland has improved since the introduction of tyrosine kinase inhibitors. However, the prognosis remains poor for frail, elderly patients with an impaired performance status.

\section{Introduction}

Approximately $20-30 \%$ of renal cell cancer (RCC) patients have distant metastases at the time of initial diagnosis [1], thus being diagnosed with synchronous metastatic renal cell carcinoma (mRCC). A limited amount of epidemiologic data on mRCC has been published, and although the safety and efficacy of modern drug therapies have been shown in randomized controlled trials, there is a scarcity of evidence on the effect of advances in medical therapy on the mRCC population. Population-based registry studies from Norway, Denmark, Sweden, Estonia, and Czech Republic [2-6] have been published during the last decade, and according to these studies, the prognosis remains relatively poor, as overall survival (OS) of only 9-14 months for the entire
mRCC population is reported in these studies. Moreover, synchronous and metachronous metastases are shown to have different prognoses, with synchronous metastatic disease tending to be of a more aggressive phenotype $[7,8]$. Whereas the role of cytoreductive nephrectomy remains controversial, clear advances in drug therapy have been made during the last 2 decades [9-11].

Until 2005, cytokine-based treatment with interferon alpha-2b (IFN- $\alpha$ ), or less frequently interleukin-2, was considered the cornerstone of drug therapy but has been subsequentially replaced with targeted therapy (TT) such as vascular endothelial growth factor (VEGF) monoclonal antibodies and VEGF receptor tyrosine kinase inhibitors (TKIs). Since phase three trials showed the superiority of sunitinib compared to IFN- $\alpha$ with tolerable side effects, TKIs 
have rapidly become the standard of care in treatment-naive clear cell mRCC, surpassing cytokine treatment $[12,13]$. The efficacy of TKIs has been confirmed in multiple studies, showing improved OS times from 18.8 up to 52 months in selected patient populations $[14,15]$.

Lately, novel immunooncologic treatments with immune checkpoint inhibitors have shown promising results and are currently gaining a robust position as an option to TKIs in first-line treatment of mRCC [16]. However, owing to the substantial cost of checkpoint inhibitors and wide clinical experience and evidence regarding TKIs, sunitinib, pazopanib, and cabozantinib still remain as the frequently used first-line treatments in Finland.

In this study, we present the patient characteristics, applied treatment protocols, and outcomes of a nationwide cohort of 977 patients with synchronous mRCC diagnosed between 2005 and 2010. Furthermore, as a rapid transition in the standard drug treatment of these patients occurred from 2007, we aimed to investigate the impact of the diagnostic time period to the survival estimate, performing separate analysis for patients diagnosed in the cytokine (2005-2007) and targeted therapy era (2008-2010).

\section{Materials and Methods}

Data from all patients with synchronous mRCC or RCC with unknown metastatic status diagnosed between 2005 and 2010 were identified from the Finnish Cancer Registry, which includes all new cancer cases in Finland. Based on these data, patient records of 2,169 consecutively diagnosed patients were requested from the according hospitals. The following numbers of patients were excluded from the analysis: 410, 500, 20, and 57 patients were diagnosed outside the defined timeframe, had no evidence of metastasis at the time of diagnosis, were under 18 years of age, and had other cancers with advanced stage, respectively. Further, 31 posthumously diagnosed cases were excluded. Also, 166 patients with insufficient data on the time of diagnosis, end of surveillance, received treatments, or metastatic stage were ruled out. In addition, due to nonrenal cell cancer histology, 2 cases of poorly differentiated urothelial carcinoma, 3 neuroendocrine/ small cell carcinomas, 1 malignant epithelioid angiomyolipoma, 1 Wilms' tumor, and 1 leiomyosarcoma were ruled out, resulting in a total number of 977 patients included in the final analysis. The following clinicopathologic variables were collected: sex, age at the time of diagnosis, primary cancer characteristics ( $\mathrm{T}$ stage, Fuhrman grade, and histology), metastasis details (location of metastasis and number of metastatic sites), Eastern Cooperative Oncology Group (ECOG) performance status, laboratory results (serum hemoglobin and C-reactive protein (CRP)), nephrectomy status, and cause of death. T stage was reassigned according to the 2017 TNM classification [17] and ECOG performance status at the time of diagnosis was evaluated retrospectively by the author if not clearly specified in the patient records.

Treatment protocols, follow-up frequency, and modality were at the discretion of the treating physician. At least one dose of the drug therapy for RCC was required to include the patient in the analyses for drug therapies.
2.1. Statistical Analysis. The main outcome, OS, was defined as the time from diagnosis to death, otherwise censored at last follow-up contact. The survival distribution and median survival were assessed with Kaplan-Meier estimates. Significance was taken at $p \leq 0.05$. Log-rank tests were used to test the influence of treatments on OS. Comparing baseline characteristics between diagnostic period groups, Pearson Chi-square test, Mann-Whitney $U$ test, and Student's $t$ test were used for categorical, ordinal, and continuous variables, respectively. Statistical analysis was performed using IBM SPSS version 26 (Chicago, IL, USA).

\section{Results}

3.1. Patient Characteristics and Biochemical and Pathological Features. Baseline characteristics are shown in Table 1. A total of 977 patients were included in the analysis: 564 (57.7\%) male and $413(42.3 \%)$ female. Mean age was 68.4 years (standard deviation (SD): 11.8 years). Mean follow-up was 22.8 months. From the 656 patients with available histological diagnosis, 584 (89.0\%) presented with clear cell histology and $72(11.0 \%)$ with other histology. Sarcomatoid changes were found in $56(8.6 \%)$ of these patients. For 321 patients, histological diagnosis was not available. Patients identified with a local tumor stage: T1, 182 (20.3\%); T2, 151 (16.9\%); T3, 401 (44.8\%); and T4, 162 (18.1\%). Additionally, T staging was not reliably defined for $81(8.3 \%)$ patients. Median primary tumor maximum diameter was 9.0 (range: 1.0-25.0) cm. Nephrectomy was performed for 518 patients. Owing to the retrospective nature of this study, serum calcium, neutrophil, and lactate dehydrogenase levels were not available for analysis for all patients; therefore, International Metastatic RCC Database Consortium (IMDC) or Memorial Sloan Kettering Cancer Center (MSKCC) risk criteria were not evaluated. However, serum hemoglobin and CRP levels were retrieved for most of the patients.

\subsection{Drug Therapies for $m R C C$ and Respective Outcomes.} Median OS was 8.80 (95\% confidence interval (CI): 7.60-10.02) months. A total number of 524 (53.8\%) patients received drug therapy. In first-line treatment, sunitinib was the most frequently used and administered to 278 (53.1\%) patients. IFN- $\alpha$ was the second most frequently used drug in a first-line setting (114 patients, $21.4 \%$ ), followed by a combination of interferon and vinblastine (58 patients, 11.1\%). All first-line treatments and respective median overall survivals are listed in Table 2. Altogether, TTs including tyrosine kinase inhibitors (TKIs: sunitinib, sorafenib, pazopanib, and regorafenib); mTORis (temsirolimus and everolimus); and VEGF inhibitor (VEGFi; bevacizumab) covered 331 (63.2\%) of first-line treatments, whereas interferon and its combinations with chemotherapy (i.e., vinblastine and capecitabine) were used for 186 patients (35.5\%). Other first-line drug therapies were single or a combination of cytotoxic chemotherapies.

3.3. Cytoreductive Nephrectomy among Patients without Drug Therapies for $m R C C$. As cytoreductive nephrectomy was a recommended treatment during the study period, the effect 
TABLE 1: Baseline characteristics of patient population according to diagnostic period.

\begin{tabular}{|c|c|c|c|c|}
\hline \multirow{3}{*}{ Baseline characteristics } & \multicolumn{3}{|c|}{ Number of patients (\%) } & \multirow{3}{*}{$p$-value } \\
\hline & \multicolumn{2}{|c|}{ Diagnostic period } & \multirow{2}{*}{ Total } & \\
\hline & $2005-2007$ & $2008-2010$ & & \\
\hline Total number of patients & 499 & 478 & 977 & \\
\hline Gender & & & & 0.094 \\
\hline M & $301(60.3 \%)$ & $263(55.0 \%)$ & $564(57.7 \%)$ & \\
\hline $\mathrm{F}$ & $198(39.7 \%)$ & $215(45.0 \%)$ & $413(42.3 \%)$ & \\
\hline Mean age at diagnosis (years) & 68.6 & 68.8 & 68.4 & 0.619 \\
\hline ECOG* & & & & $<0.001$ \\
\hline 0 & $23(5.5 \%)$ & $40(8.6 \%)$ & $63(7.2 \%)$ & \\
\hline 1 & $202(48.6 \%)$ & $233(50.3 \%)$ & $435(49.5 \%)$ & \\
\hline 2 & $103(24.8 \%)$ & $124(26.8 \%)$ & $227(25.8 \%)$ & \\
\hline 3 & $61(14.7 \%)$ & $59(12.7 \%)$ & $120(13.7 \%)$ & \\
\hline 4 & $27(6.5 \%)$ & $7(1.5 \%)$ & $34(3.9 \%)$ & \\
\hline$T$ stage* $^{*}$ & & & & 0.567 \\
\hline$T 1$ & $95(21.7 \%)$ & $87(19.0 \%)$ & $182(20.3 \%)$ & \\
\hline$T 2$ & $69(15.8 \%)$ & $82(17.9 \%)$ & $151(16.9 \%)$ & \\
\hline T3 & $191(43.6 \%)$ & $210(45.9 \%)$ & $401(44.8 \%)$ & \\
\hline$T 4$ & $83(18.9 \%)$ & $79(17.2 \%)$ & $162(18.1 \%)$ & \\
\hline$N$ stage & & & & 0.507 \\
\hline No & $305(61.1 \%)$ & $302(63.2 \%)$ & $607(62.1 \%)$ & \\
\hline N1 & $194(38.9 \%)$ & $176(36.8 \%)$ & $370(37.9 \%)$ & \\
\hline Number of metastatic sites & & & & 0.823 \\
\hline 1 & $150(30.1 \%)$ & $135(28.2 \%)$ & $285(29.2 \%)$ & \\
\hline 2 & $159(31.9 \%)$ & $156(32.6 \%)$ & $315(32.2 \%)$ & \\
\hline$\geq 3$ & $190(38.1 \%)$ & $187(39.1 \%)$ & $377(38.6 \%)$ & \\
\hline \multicolumn{5}{|l|}{ Metastatic sites } \\
\hline Distant lymph nodes & $112(22.4 \%)$ & $133(27.8 \%)$ & $245(25.1 \%)$ & 0.052 \\
\hline Lungs & $297(59.5 \%)$ & $302(63.2 \%)$ & $599(61.3 \%)$ & 0.240 \\
\hline Bone & $154(30.9 \%)$ & $159(33.3 \%)$ & $313(32.0 \%)$ & 0.421 \\
\hline Adrenal gland & $101(20.2 \%)$ & $87(18.2 \%)$ & $188(19.2 \%)$ & 0.419 \\
\hline Liver & $102(20.4 \%)$ & $96(20.1 \%)$ & $198(20.3 \%)$ & 0.890 \\
\hline Cerebral & $30(21.0 \%)$ & $25(24.0)$ & $55(22.3 \%)$ & 0.568 \\
\hline Histology tumor type ${ }^{*}$ & & & & 0.800 \\
\hline Clear cell carcinoma & $285(89.3 \%)$ & $299(88.7 \%)$ & $584(89.0 \%)$ & \\
\hline Other & $34(10.7 \%)$ & $38(11.3 \%)$ & $72(11.0 \%)$ & \\
\hline Nephrectomy & $246(49.3 \%)$ & $272(56.9 \%)$ & $518(53.0 \%)$ & 0.017 \\
\hline Hemoglobin $<$ LLN $^{*}$ & $193(60.5 \%)$ & $207(60.5 \%)$ & $400(60.5 \%)$ & 0.995 \\
\hline $\mathrm{CRP}>\mathrm{ULN}$ & $211(82.1 \%)$ & $216(74.7 \%)$ & $427(78.2 \%)$ & 0.038 \\
\hline
\end{tabular}

${ }^{*}$ Histological diagnosis was missing for 318 patients: T stage for 78 , hemoglobin for 313, CRP for 428, and ECOG status for 95 patients. The percentages in the second column were calculated only for the group of patients for whom data on these variables were available. Abbreviations: $I Q R=$ interquartile range; LLN = lower limit of normal; ULN = upper limit of normal.

of this major surgery on median OS was separately evaluated among patients who did not receive drug therapies for mRCC. Only patients with primary tumor histology available were analyzed. Cytoreductive nephrectomy, or nephrectomy and metastasectomy with curative intent, was performed for 89 and 31 patients who did not receive drug therapies for $\mathrm{mRCC}$, respectively. Ninety patients were not treated with either nephrectomy or drug therapies. Though four patients were alive at the end of the follow-up after cytoreductive nephrectomy only, the median OS after cytoreductive nephrectomy was poor: 3.88 (95\% CI 2.80-4.96) months (Figure 1). The OS for patients with no surgical or drug therapies for mRCC was 2.60 (95\% CI 1.79-3.40) months. The dismal prognosis of cytoreductive nephrectomy is at least partly explained by the fact that 43 patients died during 90 days after nephrectomy.

3.4. Time of Diagnosis as a Prognostic Factor. As the paradigm shift from interferon to TTs in standard drug therapy followed the deployment of sunitinib in 2007, we chose to separately examine cases diagnosed between 2005 and 2007, compared to 2008-2010. A total of 499 patients were diagnosed between 2005 and 2007 and 478 patients between 2008 and 2010. The median OS of the patients diagnosed at the latter era was significantly better $(11.1 ; 95 \%$ CI: 8.8-13.4 months vs. 7.0 ; 95\% CI: $5.7-8.3$ months, $p \leq 0.001$ ) (Figure 2). The proportions of patients treated with interferon and TTs and those without drug therapies for mRCC, with 
TAвLE 2: First-line drug therapies and median overall survival (OS) times with respective $95 \%$ confidence intervals (CIs).

\begin{tabular}{lcc}
\hline Drug & No. of patients (\%) & Median OS (95\% CI) (months) \\
\hline No drug therapy & $453(46.4 \%)$ & $3.0(2.6-3.4)$ \\
Sunitinib & $278(28.5 \%)$ & $20.1(15.7-24.5)$ \\
Interferon single & $112(11.5 \%)$ & $14.5(8.5-20.6)$ \\
Interferon + vinblastine & $58(5.9 \%)$ & $15.6(12.6-18.7)$ \\
Interferon + capecitabine & $16(1.6 \%)$ & $13.4(1.8-25.0)$ \\
Temsirolimus & $16(1.6 \%)$ & $10.8(8.5-13.1)$ \\
Sorafenib & $15(1.5 \%)$ & $19.9(0.0-44.5)$ \\
Pazopanib & $7(0.7 \%)$ & $19.7(16.0-23.5)$ \\
Bevacizumab & $7(0.7 \%)$ & $15.4(8.9-22.0)$ \\
Bevacizumab + interferon & $3(0.3 \%)$ & $54.5(4.1-104.8)$ \\
Regorafenib & $3(0.3 \%)$ & $51.3(0.0-125.9)$ \\
Vinblastine & $2(0.2 \%)$ & 1.5 \\
Everolimus & $1(0.1 \%)$ & 8.5 \\
Vinblastine + bevacizumab & $1(0.1 \%)$ & 9.2 \\
Etoposide & $1(0.1 \%)$ & 3.3 \\
Ifosfamide + doxorubicin & $1(0.1 \%)$ & 18.3 \\
Capecitabine & $1(0.1 \%)$ & 6.4 \\
Erlotinib & $1(0.1 \%)$ & 10.3 \\
Ifosfamide and mesna + adriamycin & $1(0.1 \%)$ & 6.1 \\
Overall & $977(100.0 \%)$ & $8.7(7.5-9.9)$ \\
\hline
\end{tabular}

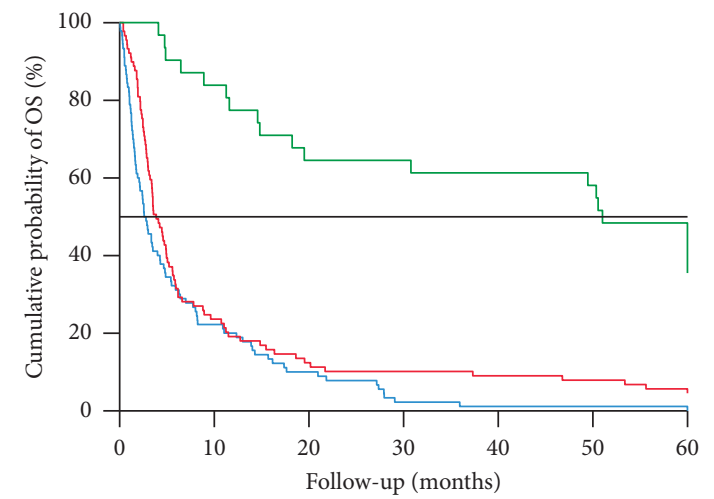

Patients at risk

No Nephrectomy 9

Cytoreductive 89

Curative 31

$$
\begin{aligned}
& \text { Nephrectomy status } \\
& \neg \text { No nephrectomy } \\
& \neg \text { Cytoreductive nephrectomy } \\
& \neg \text { Operation with curative intent }
\end{aligned}
$$

FIGURE 1: Kaplan-Meier analysis of overall survival in patients who did not receive drug therapies for histologically confirmed renal cell cancer. For this analysis, the follow-up time was limited to 60 months due to low number of patients surviving longer.

respective OS rates, are presented in Table 3. The OS tended to improve among patients treated with TTs as first-line drug therapy.

\section{Discussion}

In this study, we present a nationwide real-life cohort including all clinically diagnosed primarily metastasized renal cell cancers among adults from a six-year period.

The estimated overall survival of all patients was 8.8 (95\% CI 7.6-10.0) months. This correlates closely to results

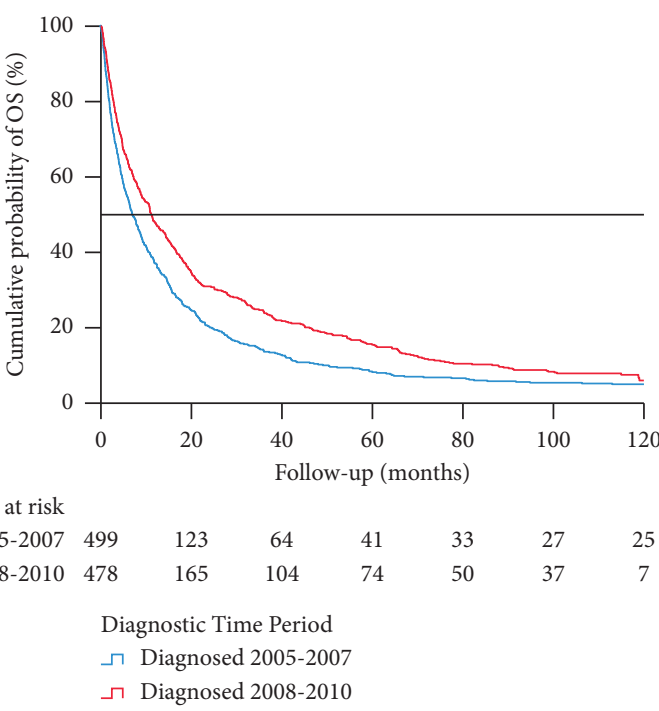

FIgURE 2: Kaplan-Meier analysis comparing overall survival of patients according to their diagnostic period (2005-2007 vs. 2008-2010).

from a Norwegian population-based study, which reported a median OS of 9.0 (95\% CI 7.9-10.1) months for all primary mRCC patients diagnosed between 2002 and 2011 [2]. A Swedish population-based study reported an OS of 12.4 (95\% CI 11.3-13.8) for mRCC patients diagnosed between 2006 and 2008, but only $31 \%$ of these patients presented with metastatic disease at initial diagnosis, which makes the data not directly comparable to ours [4]. Median OS for mRCC patients receiving drug therapies in the Danish population increased from 11.5 to 17.2 months from 2006 to 2010, but OS for untreated patients remained at 3.0 months for the same period, which is identical to our results [3]. 
TABLE 3: First-line drug therapies according to time of diagnosis. Number of patients and overall survival (OS) rates with 95\% confidence intervals (CIs) are presented. Seven patients received only chemotherapy and were excluded from data presented in this table.

\begin{tabular}{|c|c|c|c|c|c|}
\hline \multirow{3}{*}{ First-line drug therapy } & \multicolumn{4}{|c|}{ Time of diagnosis } & \multirow{3}{*}{$\begin{array}{c}\text { Total } \\
N=970 \\
n(\%)\end{array}$} \\
\hline & \multicolumn{2}{|c|}{$2005-2007(N=494)$} & \multicolumn{2}{|c|}{$2008-2010(N=476)$} & \\
\hline & $n(\%)$ & OS $(95 \% \mathrm{CI})$ & $n(\%)$ & OS $(95 \% \mathrm{CI})$ & \\
\hline No medical treatment & $260(52.1)$ & $2.86(2.22-3.50)$ & $193(40.4)$ & $3.38(2.77-4.00)$ & $453(46.4)$ \\
\hline Interferon \pm chemotherapy & $169(33.9)$ & $15.3(12.5-18.1)$ & $17(3.6)$ & $13.2(4.74-21.6)$ & $186(19.0)$ \\
\hline $\mathrm{TT}$ & $65(13.0)$ & $14.8(11.9-17.6)$ & $266(55.6)$ & $20.2(16.3-24.1)$ & $331(33.9)$ \\
\hline
\end{tabular}

A significant proportion of cases represented a frail elderly population with reduced overall health status and limited tolerance of surgical treatment or drug therapies for mRCC. Although an age $\geq 75$ years has not been proven as an independent prognostic factor for mRCC patients [18], it explains the relatively poor overall survival compared to various mRCC studies devoted to histologically confirmed clear cell tumors in patients with relatively good (ECOG $0-1$ ) performance status. Cytoreductive nephrectomy as the only treatment resulted in no OS advantage. Almost half of the patients, who did not receive drug therapies for $\mathrm{mRCC}$, deceased during 90 days after nephrectomy. However, single patients had excellent prognosis after cytoreductive nephrectomy only, which may be explained by spontaneous regression of the metastases [19-21], or incorrect radiological diagnosis of mRCC. Cytoreductive nephrectomy is major surgery especially in locally advanced cases, and the patients should fit well for surgery.

The OS observed in the first-line sunitinib group in our unselected patient population was 20.1; 95\% CI: 15.7-24.5 months, which is comparable to median OS of 18.4 months reported in an expanded-access sunitinib trial published in 2009 , including $7 \%$ patients with brain metastases, $13 \%$ with an ECOG performance status of 2 or worse, and 13\% nonclear cell RCC, presenting a more diverse patient population [22]. In comparison, a median OS of 28.6 months was reported in a recent retrospective analysis including clear cell mRCC patients from all IMDC risk groups treated with first-line sunitinib, noting a prominent difference in the median OS of IMDC favorable (52.1, 95\%CI: 43.4-61.2 months) vs. combined IMDC intermediate and poor (23.2, 95\% CI: 21.0-25.8 months) groups [15].

Among the patients with a poorer performance status, histological diagnosis was also frequently missing. This resulted mostly from the fact that acquiring histology was not judged to be essential in cases with absence of effective surgical or drug therapy options for mRCC, due to the poor general health of the patient. However, a proportion of patients received drug therapies for $\mathrm{mRCC}$ despite lacking histological diagnosis (data not shown).

In the pivotal phase three sunitinib vs. interferon trial by Motzer et al. [12], the median progression-free survival was significantly longer in the sunitinib group compared to the interferon group (11 vs. 5 months, respectively). Subsequently, a significant OS benefit for the sunitinib group (26.4 vs. 21.8 months) compared to the IFN- $\alpha$ group was reported [13]. In our investigation, a similar trend of OS difference in sunitinib and IFN- $\alpha$ groups (20.1 and 14.5 months, respectively) was observed; however, this remains statistically insignificant.

We found that the overall survival prognosis of mRCC improved significantly during the observed period, which upholds the similar findings from other population-based studies [2-6]. We found a significant difference in the baseline factors ECOG and increased CRP value between diagnostic time groups in favour of the latter era, but no statistically significant difference in the number or location of metastatic sites or other baseline factors (Table 1). Thus, the data suggests that the overall treatment of the more recently diagnosed patient population has been more effective. The transition of primary first-line drug therapy from interferon-based regimes to TT and simultaneous improvement of OS can be observed in Table 3 and Figure 2, respectively.

The greatest strength of our study is that it is based on the data from the Finnish Cancer Registry, which receives a notification of every suspected or diagnosed cancer in Finland directly from the treating hospital [23]. Based on this information, complementary patient record acquisitions from the hospitals were made to form a comprehensive review of the available patient data, describing the national situation as accurately as possible. The patient records were thoroughly examined by the author, collecting all the planned information that was available. In addition to the effects of drug therapies in a large real-life cohort, this study brings forth valuable and reliable information on the natural history of synchronous mRCC, which is not a widely covered subject in the current epidemiologic literature.

However, our study has obvious limitations. It is a retrospective study, and there are some proportions of missing data. Particularly, laboratory results were not comprehensively available in many cases, probably because they were not included in the clinical practice at that time. We were not able to retrieve the original diagnostic imaging material for reevaluation but relied on the local radiology reports on assessing the diagnostic stage and radiologic progression or treatment response. In the present study, if ECOG performance status was not assessed and recorded at diagnosis, it was assigned retrospectively by the author, when judged possible based on the available information. It would be of great interest to evaluate the effect of novel immunooncological therapies among this patients population, but due to financial issues, the use of this treatment modality is just expanding in Finland at the moment. So the perfect time for this analysis is yet to come. 


\section{Conclusion}

A rapid change of treatment of choice from cytokines to TT occurred in Finland between 2007 and 2008. This resulted in a significant improvement in OS of mRCC patients. Our findings suggest that cytoreductive nephrectomy should be performed only for patients not likely to waste away after surgery. A significant proportion of mRCC patients are diagnosed in a late symptomatic phase of the disease, and for such patients, the overall survival remains poor despite the advancements in cancer therapy.

\section{Data Availability}

Restrictions apply to the availability of these data, which were used under license for the current study, and so are not publicly available. Data are, however, available from the authors upon reasonable request.

\section{Ethical Approval}

The local ethics council of Oulu University Hospital (Oulu, Finland) approved the present study, which was conducted according to the Declaration of Helsinki.

\section{Consent}

Informed written consent from included study patients was not obtained, for the National Institute for Health and Welfare (Helsinki, Finland) approved the present study and the use of registry data following local ethical approval, according to Finnish law. Patient data were anonymised and deidentified prior to statistical analysis.

\section{Conflicts of Interest}

The authors have no conflicts of interest to declare.

\section{Authors' Contributions}

All the authors contributed to the study conception and design. Material preparation and data collection were performed by Lauri Laru and analyses were performed by Lauri Laru and Markku Vaarala. The first draft of the manuscript was written by Lauri Laru and all authors commented on previous versions of the manuscript. All authors read and approved the final manuscript.

\section{Acknowledgments}

The authors would like to thank Pasi Ohtonen (M.Sc.) for assistance with statistical analysis and Kaisa Makkonen (MD) for assistance on screening and analyzing the patient records. In addition, the authors would like to thank Ms. Tuula Lähtevänoja, Ms. Katja Vaihoja, and Ms. Leena Heikkilä (Oulu University Hospital, Oulu, Finland) for acquisition of the patient records. This work was supported in part by the Finnish Urological Association to Lauri Laru.

\section{References}

[1] B. Ljungberg, S. C. Campbell, H. Y. Cho et al., "The epidemiology of renal cell carcinoma," European Urology, vol. 60, no. 4, pp. 615-621, 2011.

[2] C. Beisland, T. Johannesen, O. Klepp et al., "Overall survival in renal cell carcinoma after introduction of targeted therapies: a Norwegian population-based study," OncoTargets and Therapy, vol. Volume 10, pp. 371-385, 2017.

[3] A. V. Soerensen, F. Donskov, G. G. Hermann et al., "Improved overall survival after implementation of targeted therapy for patients with metastatic renal cell carcinoma: results from the Danish renal cancer group (DARENCA) study-2," European Journal of Cancer, vol. 50, no. 3, pp. 553-562, 2014.

[4] T. Wahlgren, U. Harmenberg, P. Sandström et al., "Treatment and overall survival in renal cell carcinoma: a Swedish population-based study (2000-2008)," British Journal of Cancer, vol. 108, no. 7, pp. 1541-1549, 2013.

[5] H. Jürgens, K. Ojamaa, H. Pokker, K. Innos, and P. Padrik, "Changes in therapy and survival of metastatic renal cell carcinoma in Estonia," BMC Cancer, vol. 20, no. 1, p. 201, 2020.

[6] A. Poprach, Z. Bortlíček, T. Büchler et al., "Patients with advanced and metastatic renal cell carcinoma treated with targeted therapy in the Czech Republic: twenty cancer centres, six agents, one database," Medical Oncology, vol. 29, no. 5, pp. 3314-3320, 2012.

[7] S. H. Kim, D.-e. Lee, B. Park et al., "Survival of patients receiving systematic therapy for metachronous or synchronous metastatic renal cell carcinoma: a retrospective analysis," BMC Cancer, vol. 19, no. 1, p. 688, 2019.

[8] S.-F. Kammerer-Jacquet, A. Brunot, A. Pladys et al., "Synchronous metastatic clear-cell renal cell carcinoma: a distinct morphologic, immunohistochemical, and molecular phenotype," Clinical Genitourinary Cancer, vol. 15, no. 1, pp. e1-e7, 2017.

[9] D. Y. C. Heng, J. C. Wells, B. I. Rini et al., "Cytoreductive nephrectomy in patients with synchronous metastases from renal cell carcinoma: results from the international metastatic renal cell carcinoma database consortium," European Urology, vol. 66, no. 4, pp. 704-710, 2014.

[10] R. C. Flanigan, S. E. Salmon, B. A. Blumenstein et al., "Nephrectomy followed by interferon alfa- $2 \mathrm{~b}$ compared with interferon alfa-2b alone for metastatic renal-cell cancer," New England Journal of Medicine, vol. 345, no. 23, pp. 1655-1659, 2001.

[11] A. Méjean, A. Ravaud, S. Thezenas et al., "Sunitinib alone or after nephrectomy in metastatic renal-cell carcinoma," New England Journal of Medicine, vol. 379, no. 5, pp. 417-427, 2018.

[12] R. J. Motzer, T. E. Hutson, P. Tomczak et al., "Sunitinib versus interferon alfa in metastatic renal-cell carcinoma," New England Journal of Medicine, vol. 356, no. 2, pp. 115-124, 2007.

[13] R. J. Motzer, T. E. Hutson, P. Tomczak et al., "Overall survival and updated results for sunitinib compared with interferon alfa in patients with metastatic renal cell carcinoma," Journal of Clinical Oncology, vol. 27, no. 22, pp. 3584-3590, 2009.

[14] D. Y. Heng, W. Xie, M. M. Regan et al., "External validation and comparison with other models of the international metastatic renal-cell carcinoma database consortium prognostic model: a population-based study," The Lancet Oncology, vol. 14, no. 2, pp. 141-148, 2013.

[15] M. F. Savard, J. C. Wells, J. Graham et al., "Real-world assessment of clinical outcomes among first-line sunitinib 
patients with clear cell metastatic renal cell carcinoma (mRCC) by the international $\mathrm{mRCC}$ database consortium risk group," The Oncologist, vol. 25, no. 5, pp. 422-430, 2020.

[16] R. J. Motzer, N. M. Tannir, D. F. McDermott et al., "Nivolumab plus ipilimumab versus sunitinib in advanced renalcell carcinoma," New England Journal of Medicine, vol. 378, no. 14, pp. 1277-1290, 2018.

[17] M. B. Amin and S. B. Edge, AJCC Cancer Staging Manual, Springer, New York, NY, USA, 8th edition, 2017.

[18] H. K. Khambati, T. K. Choueiri, C. K. Kollmannsberger et al., "Efficacy of targeted therapy for metastatic renal cell carcinoma in the elderly patient population," Clinical Genitourinary Cancer, vol. 12, no. 5, pp. 354-358, 2014.

[19] L. B. E. Shields and A. Rezazadeh Kalebasty, "Spontaneous regression of delayed pulmonary and mediastinal metastases from clear cell renal cell carcinoma," Case Reports in Oncology, vol. 13, no. 3, pp. 1285-1294, 2020.

[20] K. Muraoka, Y. Nishikawa, and T. Isoyama, "Spontaneous regression of metastatic renal cell carcinoma after cytoreductive nephrectomy: a case report," Urology Case Reports, vol. 31, Article ID 101179, 2020.

[21] K. Ueda, S. Suekane, T. Mitani et al., "Spontaneous regression of multiple pulmonary nodules in a patient with unclassified renal cell carcinoma following laparoscopic partial nephrectomy: a case report," Molecular and Clinical Oncology, vol. 5, no. 1, pp. 49-52, 2016.

[22] M. E. Gore, C. Szczylik, C. Porta et al., "Safety and efficacy of sunitinib for metastatic renal-cell carcinoma: an expandedaccess trial," The Lancet Oncology, vol. 10, no. 8, pp. 757-763, 2009.

[23] L. Teppo, E. Pukkala, and M. Lehtonen, "Data quality and quality control of a population-based cancer registry: experience in Finland," Acta Oncologica, vol. 33, no. 4, pp. 365-369, 1994. 